\title{
Density, Alternative Determinant of Star Formation Efficiency of the Milky Way GMCs: Core Reason for Low Star Formation Efficiency
}

\author{
Okezuonu Patrick Chinedu, Ogwo Jemima Ngozi \\ Department of Industrial Physics, Faculty of Physical Science, Abia State University, Uturu, Nigeria \\ Email address: \\ chineduokezuonupatrick@gmail.com (O. P. Chinedu),jn.ogwo@abiastateuniversity.edu.ng (O. J. Ngozi) \\ To cite this article: \\ Okezuonu Patrick Chinedu., Ogwo Jemima Ngozi. Density, Alternative Determinant of Star Formation Efficiency of the Milky Way GMCs: \\ Core Reason for Low Star Formation Efficiency. International Journal of Astrophysics and Space Science. Vol. 9, No. 3, 2021, pp. 45-50. \\ doi: 10.11648/j.ijass.20210903.12
}

Received: October 11, 2021; Accepted: November 5, 2021; Published: November 24, 2021

\begin{abstract}
The overall efficiency with which Milky Way Giant Molecular Clouds (GMCs) is forming stars was determined by deriving an equation using density of cloud (i.e. stellar density/ total cloud density), which is the core parameter that determines star formation other than the mass of cloud, and comparing with mass (i.e. stellar mass/ total gas mass) as was propounded by previous researchers, to ascertain the reasons the observed star formation efficiency of Milky Way Giant Molecular Clouds $\left(\epsilon_{\mathrm{GMC}}\right)$ is low. This will aid understanding the physical factors behind the formation of stars from interstellar gas and develop a predictive theory of star formation and evolution of galaxies. A total of 191 star formation complexes-giant molecular cloud (SFC-GMC) complexes was used in estimating the following cloud parameters: density as 93.8218 solar mass/parsec squared, average stellar density as 2.67872 solar mass/parsec squared, average luminosity as 9.87E24 solar luminosity, average effective temperature as 498,647 solar temperature, average stellar radius as 51.4522 parsec and average cloud radius as 325507 parsec as well as the total mass in stars $M_{\star}$ harbored by the individual clouds (20,831 solar mass), which was inferred from Wilkinson Microwave Anisotropy probe (WMAP) free-free. Finally, the overall efficiency with which Milky Way Giant Molecular Clouds is forming star gave 0.0289573 which is less than the previous estimate as 0.030849 , showing that not all the masses of the cloud were present at the end of the star formation, and this reduction in mass are caused by magnetic field, supersonic turbulence, self-regulation and unbound states of its internal structure, which are the reasons the observed star formation efficiencies are low.
\end{abstract}

Keywords: Galaxies, Star Formation Efficiency, Cloud Density, Stellar Mass, Gas Mass

\section{Introduction}

Everything known about galaxy, such as its observations, definition etc. is through its stellar contents. Therefore, any theory of galaxy formation has to start by addressing how stars' form, the main processes that drive star formation and what mass fraction of a gas cloud can be converted into stars (star formation efficiency (SFE or $\epsilon$ )). Star formation on the other hand is the process by which interstellar medium of gas and dust collapse and form stars in its dense region known as molecular clouds or star-forming region [24].

"Lee et al and Murray N. defined star formation efficiency of the GMCs $\left(\epsilon_{G M C S}\right)$ as the fraction of the GMC that is converted into stars over the lifetime of the GMCs [16, 22]". Mathematically $\epsilon_{G M C s}$ is defined as the total mass in stars, produced over the life time of the GMC, divided by the overall GMC mass which also includes the total mass in those stars

$$
\epsilon_{G M C S}=\frac{M_{\star}}{M_{G M C}+M_{\star}}=\frac{M_{\star}}{M_{g}}
$$

where $M_{\star}$ is the total mass of the individual stars inside the clouds, $M_{G M C}$ is the total mass of the remaining cloud that does not finally convert to star and $M_{g}$ is the total mass of the cloud.

After thorough examination on this parameter (Mass) found in equation (1), it was found that using mass to calculate the efficiency of clouds in forming stars cannot provide the exact value for the prescribed efficiency because some of these masses of the clouds have been destroyed by both internal and 
external forces such as magnetic flux of the internal structure of the clouds [7, 13], supersonic turbulence and self-regulation, over a long period of time [4, 12 20,] (i.e. from the time of gravitational collapse to the time the cloud is ready to form stars). Hence, making $M_{g}$ of these clouds not ideal for $\epsilon$, rather $\sum_{g}[20]$.

$$
\epsilon_{G M C s}=\frac{\Sigma_{\star}}{\Sigma_{G M C}+\Sigma_{\star}}=\frac{\Sigma_{\star}}{\Sigma_{g}}
$$

Grudic et al defied SFE, as the fraction of the gas mass that is converted to stars across the entire life time of a cloud [11]:

$$
\epsilon_{\text {int }}=\frac{M_{\star(t=\infty)}}{M_{\operatorname{gas}(t=0)}}
$$

where $M_{\star}$ is the total mass in stars formed and $M_{\text {gas }}$ is the total gas mass. $\epsilon_{\text {int }}$ is of particular interest because it is sensitive to the details of stellar feedback physics, as eventually a sufficient number of massive stars will form to expel the gas. $\epsilon_{\text {int }}$ ultimately determines the mapping between the GMC and star cluster mass functions in a galaxy, and dictates the fraction of stars remaining in a gravitationally bound clusters after gas expulsion. The inferred gas mass will also vary over the lifetime of the cloud: while the effect of gas consumption might be negligible if overall efficiencies are small, molecular gas will also be destroyed and ejected by stellar feedback, so towards the end of a cloud's star forming lifetime the inferred efficiency might be biased upward [10].

As already known [23], dense gas $\left(\geq 10^{5} \mathrm{~cm}^{-3}\right)$ has a great impact on star formation in molecular clouds. At times, cores of very dense gas resist the disruptive forces of winds and radiations from nearby newly-formed stars and they help to maintain their ongoing star formation even in hostile environments. Therefore, the sample of the dense region of these clouds is generally small compared to the total masses of these clouds.

Many works have been done on SFE (e.g. [17, 19] etc.) of the Giant Molecular Clouds (GMCs) and it has been found that these $\epsilon$ are so low and the reasons behind this are yet concluded.

This research work was carried out to ascertain the reasons for low star formation efficiencies of the GMCs and is organized as follows: section 2 focused with the internal and external forces responsible for the destruction of the cloud masses, section 3 is the methodology used in the estimations, section 4 results are presented, section 5 discussions of results, and finally, section 6 , conclusion and our contribution to knowledge.

\section{The Disruptive Forces}

There are so many forces responsible for the low star formation efficiencies, and these forces affects the masses of the clouds in a greater percentage than densities which is the core determinant, necessary for a cloud to form star (s). These forces such as forces of stellar winds and radiations from newly formed stars, (stellar feedback), magnetic flux, supersonic turbulence etc. $[2,8,10]$, etc. are part of the reasons the star formation efficiencies are so low and at the same time sources of trigger for star formation.

\subsection{Stellar Feedback}

Stellar Feedback may not only be important for promoting star formation under certain conditions. For instance, star formation in one place may be induced by the feedback of starformation activity in other places, which is called an "induced" star formation, i.e. the density and velocity structure of the ISM, are actually produced by stars themselves. Even if the large-scale star formation environments are produced by the other processes described above, compression by energy feedback from nearby stars may play an important role in triggering star formation in pre-existing clouds [9]. Sellar Feedback may also terminate or prevent star formation; it also destroys some parts of the already existing cloud thereby reducing the $M_{g}$ of the cloud.

\subsection{Self-Regulation}

The third mechanism that may be important for regulating the overall $\epsilon_{G M C S}$ is self-regulation that is the actual process of star formation itself which destroy the molecular clouds, and hence regulates the $\epsilon_{G M C S}$; in particular. Feedback from protostellar winds is believed to play an important role in regulating the overall $\epsilon_{G M C s}$ of protostellar cores [18].

\subsection{Supersonic Turbulence}

In a self-gravitating turbulent medium, the collapse of gas clouds can be affected by turbulence in two different ways. First, because turbulent motion increases the effective velocity dispersion of the gas can delay or suppress gravitational collapse. Second, shocks produced by the supersonic flow in a turbulent medium can promote gravitational collapse by increasing the gas density.

\subsection{Magnetic Flux}

A major question to understand the role of the magnetic field in molecular cloud evolution is how it correlates with the other fields and in particular with the density, [12]. Owing to that he stated that the mean magnetic intensity is independent of gas density, n, for values up to about 300 $\mathrm{cm}^{-3}$. At higher densities, that is to say at least up to $10^{6-7}$ $\mathrm{cm}^{-3}$, the mean magnetic intensity has been found to increase with $\mathrm{n}$ broadly like a power-law, that is $\mathrm{B} \simeq n^{k}$. The exact value of $\mathrm{k}$ is still a matter of debate. Earlier works [5] obtained $\mathrm{k}=1 / 2$ but more elaborated Bayesian analysis led to $\mathrm{k} \simeq 0.65$ [6]. Finally, the mass to magnetic flux ratio, $M / \Phi$, can be estimated by combining the column density of the observed component along the line of sight and the observed magnetic intensity, leading to the conclusion that the atomic and diffuse molecular gas is subcritical, that is to say dominated by the magnetic field, while dense regions, such as dense cores, are generally supercritical.

Prior, to our understanding of galaxy formation, the cold gas in a star-forming galaxy is assembled hierarchically through the cooking and accretion of gas in merging dark matter halos. This process produces high levels of turbulence 
in the gas. Also cosmological numerical simulations of galaxy disk formation show that gravitational instabilities can generate high level of turbulence [3, 14, 20].

\section{Methods}

The first step in the calculation of $\epsilon_{G M C}$ is to identify the host GMCs of the SFCs, [15]. The cloud catalog provides the $\Sigma_{G M C}$ (cloud density) estimate, and is presented partially in table 1 and fully in appendix A.

\subsection{Estimation of Stellar Mass from Free-Free Flux}

The average mass of stars associated with each cloud is evaluated from the WMAP free-free fluxes. For a given WMAP free-free source (the large $\sim 1^{\circ}-2^{\circ}$ wide regions identified by their peak free-free flux in WMAP; see [21] for more detail), aperture photometry was first performed to compute the total free-free flux $F_{v}$. The total flux is divided into constituent star formation complexes ((SFCs) and by extension, constituent SFC-GMC complexes), proportional to the relative SFC $8 \mu \mathrm{m}$ fluxes computed from Spitzer GLIMPSE and MSX images. The mass of "live" stars is given by the formula

$$
M_{\star}=Q /\left(\left\langle M_{\star}\right\rangle /\langle q\rangle\right)
$$

$$
\text { where }\left(\left\langle M_{\star}\right\rangle /\langle q\rangle\right)=6.3 \times 10^{46} s^{-1} M^{-1}
$$

Having assigned fluxes $F_{v}$ to each SFC, kinematic distance $D$ was used to calculate the free-free luminosity

$$
L_{v}=4 \pi D^{2} f_{v}
$$

emitted by that region, and the rate

$$
\mathrm{Q}=1.37 \times 10^{26} L_{v} S^{-1}
$$

$\mathrm{Q}$ is the ionizing photons emitted by each source per second required to power the observed free-free [21].
Following [25], the rate of ionizing photons (Q) was increase by a factor 1.37 to account for the fact that some of those photons are absorbed by dust, and hence do not contribute to the free-free emission detected by WMAP. The live stellar mass was then calculated using Equation (4) above.

\subsection{Estimation of Stellar Densities from Free-Free Flux and Other Clouds Parameters}

At the same time, stellar density of the individual cloud was calculated using mass-density relation, given as:

$$
\Sigma_{\star}=\frac{M_{\star}}{V_{\star}}
$$

Where $V_{\star}$ is the volume of the individual stars, and is given as $4 / 3 \pi R_{\star}^{3}$.

The effective temperature $T_{\text {eff }}$ was estimated using temperature-luminosity relation given as $T=\left(L_{v}^{b r} / 4 \pi R^{2} \sigma_{S B}\right)^{0.25}$, and finally, the radius of the individual stars $\left(R_{\star}\right)$ and clouds $\left(R_{\text {cloud }}\right)$ was estimated using $\mathrm{R}=M^{0.7}$ as shown in table 1 , where $\sigma_{S B}$ is the StephanBoltzman constant $=5.6696 \times 10^{-8} \mathrm{kgs}^{-3} \mathrm{~K}^{-4}$. Finally, having selected the GMCs and calculated its cloud parameters, the $\epsilon_{G M C}$ was then estimated by substituting into equation (2).

\section{Results}

The results of 191 selected GMCs were presented, with their cloud properties such as: total gas masses $\left(M_{g}\right)$, average stellar masses $\left(M_{\star}\right)$, Luminosities $\left(L_{v}\right)$, Effective Temperature

\begin{tabular}{|c|c|c|c|c|c|c|c|c|c|c|c|c|}
\hline $\mathbf{S} / \mathbf{N}$ & $\begin{array}{l}\text { GMCs } \\
\text { no } \\
\end{array}$ & $\mathrm{Q} s^{-1}$ & $\begin{array}{l}M_{g} \\
M_{\odot} \\
\end{array}$ & $\begin{array}{l}M_{g} \\
M_{\odot} \\
\end{array}$ & $\begin{array}{l}\Sigma_{G M C} \\
M_{\odot} / p c^{2}\end{array}$ & $\begin{array}{l}\Sigma_{G M C} \\
M_{\odot} / p c^{2}\end{array}$ & $\begin{array}{l}M_{G M C} \\
M_{\odot} \\
\end{array}$ & $\begin{array}{l}L_{v} \\
\operatorname{ergs}^{-1} H z^{-1}\end{array}$ & $\operatorname{Rad} p c$ & $\begin{array}{l}\mathbf{T} \\
T_{\odot} \\
\end{array}$ & $\begin{array}{l}\text { SFE } M_{g} \\
\text { GMCs } \\
\end{array}$ & $\begin{array}{l}\text { SFE DEN } \\
\text { GMCs } \\
\end{array}$ \\
\hline 1 & 1726 & $64000 \mathrm{E}+05$ & 577778 & 947000 & $6.31 \mathrm{E}+01$ & 0.757098 & 369222 & $2.73684 \mathrm{E}+26$ & 15256.2 & 1133405 & 0.610114 & 0.110260 \\
\hline 2 & 1761 & $1.65000 \mathrm{E}+52$ & 261905 & 8490000 & $1.61 \mathrm{E}+02$ & 1.198825 & 8228095 & $1.24060 \mathrm{E}+26$ & 70833.0 & 431604 & 0.030849 & 0.047974 \\
\hline 3 & 26 & $9.50000 \mathrm{E}+51$ & 150794 & 2970000 & $2.23 \mathrm{E}+02$ & 3.027471 & 2819206 & $7.14286 \mathrm{E}+25$ & 33957.0 & 542998 & 0.050772 & 0.019571 \\
\hline 4 & 279 & $8.28000 \mathrm{E}+51$ & 131429 & 3510000 & $2.18 \mathrm{E}+02$ & 2.381247 & 3378571 & $6.22556 \mathrm{E}+25$ & 38169.4 & 494860 & 0.037444 & 0.319713 \\
\hline 5 & 440 & $7.24000 \mathrm{E}+51$ & 114921 & 2330000 & $1.51 \mathrm{E}+02$ & 2.093327 & 2215079 & $5.44361 \mathrm{E}+25$ & 28651.6 & 552322 & 0.049322 & 0.003108 \\
\hline 6 & 171 & $7.00000 \mathrm{E}+51$ & 111111 & 769000 & $1.13 \mathrm{E}+02$ & 2.410169 & 657889 & $5.26316 \mathrm{E}+25$ & 13187.1 & 807296 & 0.144488 & 0.015906 \\
\hline 8 & 583 & $6.04000 \mathrm{E}+51$ & 95873 & 3430000 & $1.88 \mathrm{E}+02$ & 2.085155 & 3334127 & $4.54135 \mathrm{E}+25$ & 37558.3 & 461040 & 0.027951 & 0.222916 \\
\hline 9 & 1054 & $5.81000 \mathrm{E}+51$ & 92222 & 1240000 & $8.71 \mathrm{E}+01$ & 1.999706 & 1147778 & $4.36842 \mathrm{E}+25$ & 18424.5 & 651897 & 0.074373 & 0.038168 \\
\hline 10 & 678 & $5.79000 \mathrm{E}+51$ & 91905 & 2690000 & $2.34 \mathrm{E}+02$ & 3.327404 & 2598095 & $4.35338 \mathrm{E}+25$ & 31683.0 & 496694 & 0.034165 & 0.019557 \\
\hline
\end{tabular}
$\left(T_{\text {eff }}\right)$, Cloud Radius (Rad), total densities $\left(\sum_{G M C S}\right)$ and star formation efficiencies $(\in)$ of the selected GMCs which we presented partially in table 1 and fully in appendix A.

Table 1. 191 Selected GMCs Efficiencies and its Estimated Cloud Parameters.

Efficiencies of Milky Way star-forming complexes and their host GMCs. (Column 2) GMCs number of Lee et al., (2016). The rest of the columns give star formation complex properties; (Column 3), ionizing photons emitted per second $Q$ (Column 4), stellar mass $M_{\star}$ (Column 5), GMC mass $M_{g}$ (Column 6) cloud densities, (column 7) the mass of GMCs that does not form stars $M_{G M C}$, (column 8) free-free luminosity $L_{v}$, (column 9) GMC radius Rad pc, (column 10) effective Temperature $T_{\text {eff }}$ and (column 11 and 12) GMC efficiency $\in$.

\section{Discussion}

This work was aimed at estimating the overall efficiency with which Milky Way GMC form stars and the factors responsible for low SFE of Milky Way GMCs.

From these result, the overall $\epsilon_{G M C}$ is 0.024832 , with standard deviation equal 0.05613 , then comparing this result with the one gotten from $M_{\star} / M_{g}$, and it was found that their outcome are closely related, which means one can also 
calculate the $\epsilon_{G M C}$ by using both equations or expressions as illustrated in [1], they considered "all the gas above the transition density to be dense "self-gravitating gas" and all the gas in the lognormal portion to be "diffuse unbound molecular gas". They also predicted that self-gravitating gas fraction $\left(f_{\text {dense }}\right)$ can then be related to a star formation efficiency which tells that the overall fraction of gas mass available for star formation at a given time based on the slope of the density PDF and the cloud environment".

The graph of $M_{\star}$ against $M_{g}$ and $\Sigma_{\star}$ against $\sum_{g}$ see figures ( 1 and 2 respectively) were plotted to check if our results are consistent with the results of the previous researchers and it was found that majority of these efficiencies clustered at a low scale between zero (0) to $100,000 M$ on $M_{\star}$ axis and zero (0) to 2,000,000M on $M_{g}$ axis (figure 1) and between zero (0) to $5 \mathrm{M} / \mathrm{pc}^{2}$ on $\Sigma_{\star}$ axis and between zero (0) to 200 $M / \mathrm{pc} 2$ on $\sum_{g}$ axis (figure 2) showing the low efficiencies of GMCs to form star.

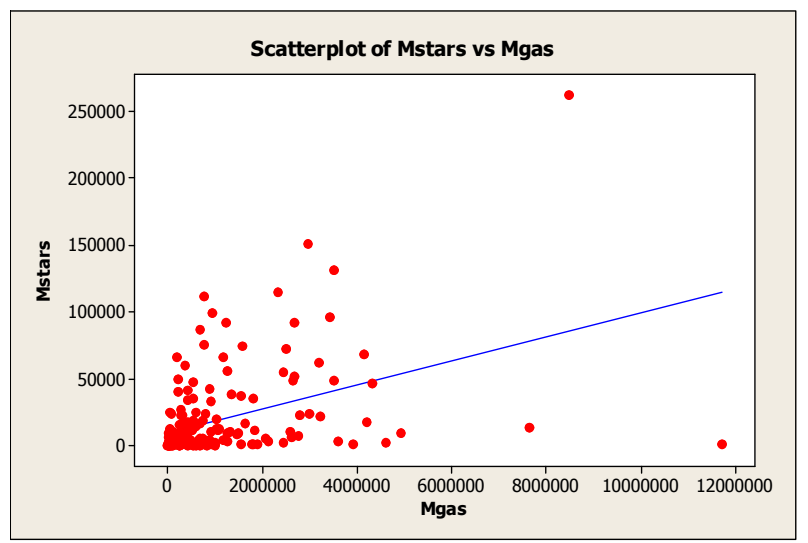

Figure 1. The scatter plot of the calculated $M_{\star} v s M_{g}$ of the GMCs in Milky Way.

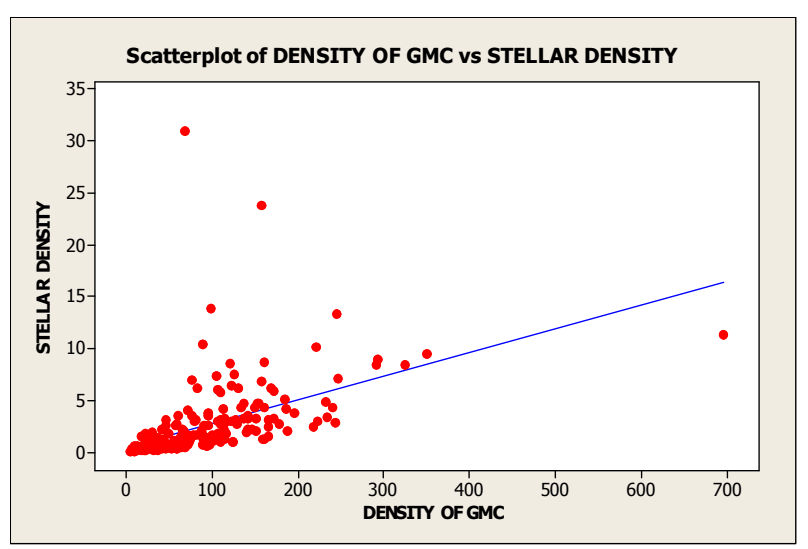

Figure 2. The scatter plot of $M_{\star}$ vs $\sum$ of the selected GMCs in Milky Way.

It was also found that the $\epsilon_{G M C}$ does not depend on $M_{g}$, as shown by the Asymptotic decay curve of our plot on Figure 3, (this means that at an interval of zero (0) to about 1,000,000 solar mass on a horizontal axis of $M_{g}$, the $\epsilon_{G M C}$ increases and above this mass it decays exponentially) rather, it depended on $M_{\star}$ which in turn depended on the $\sum_{g}$ as shown in Figures 4.

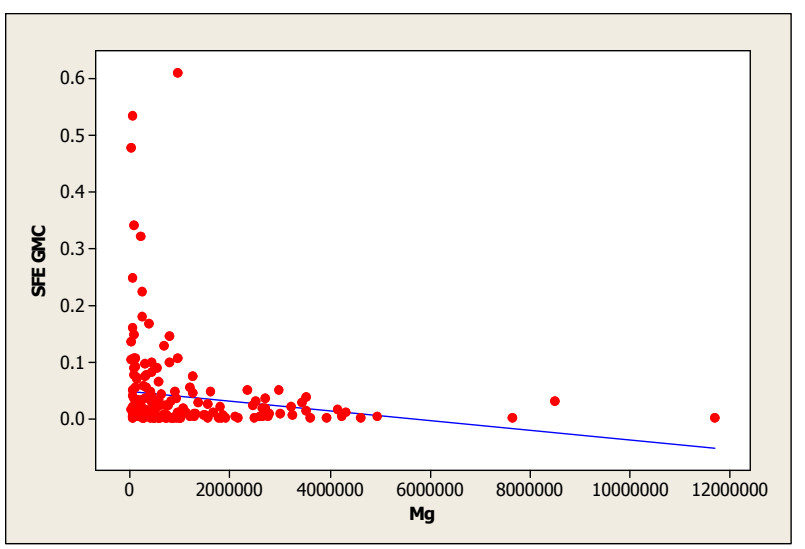

Figure 3. The scatter plot of SFE-GMC vs $M_{g}$ of the selected GMCs in Milky.

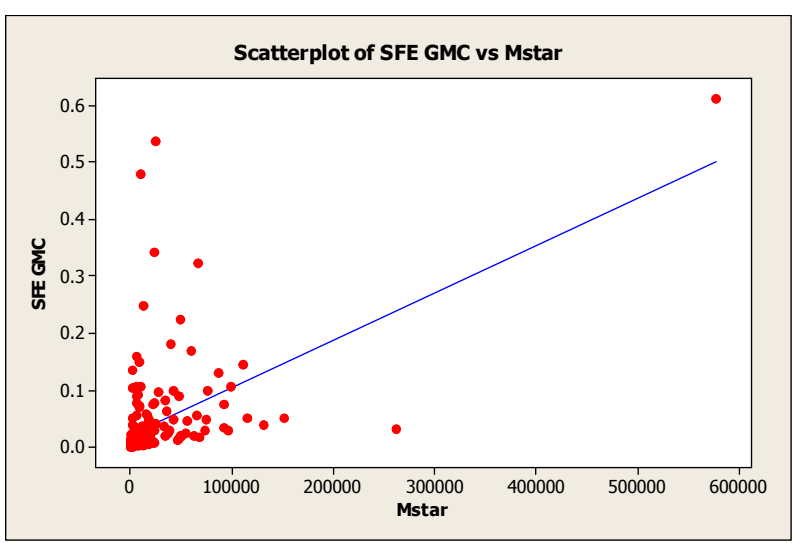

Figure 4. The scatter plot of SFE-GMC vs $M_{g}$ of the selected GMCs in Milky.

This is in line with [4] who said that the unbound clouds can naturally result in lower SFEs than their bound (dense) counterparts, simply because the gas is unable to rid itself of the excess kinetic energy and achieve a bound state. It was concluded that what determines the state and the efficiency of a cloud to form star is its density.

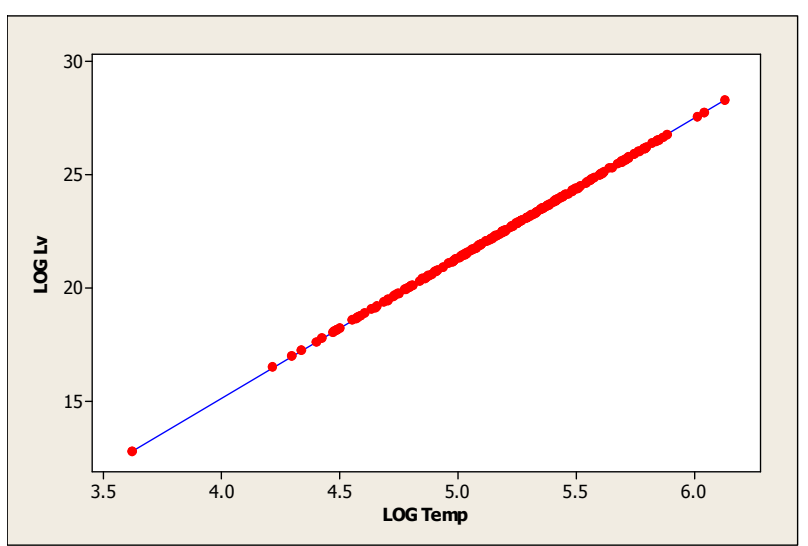

Figure 5. The scatter plot of Luminosity vs Temperature of the selected GMCs in Milky Way.

Other cloud parameters such as $\log L_{v}$ vs $\log T_{\text {eff }}$ Figure 5, $\log L_{v}$ vs $\log \operatorname{Rad}$ Figure 6 etc was plotted. to check the correlation of parameters toward another. This was to ascertain if some of the formulae used here relate to another or not, but the outcome shows tight correlations: i.e. mass- 
luminosity relation $\left(\mathrm{L}=M^{3.9} \approx 4\right)$, mass - radius relation $\left(\mathrm{R}=M^{0.7}\right)$ and luminosity-temperature relation $(\mathrm{T}=$ $\left.\left(\frac{L_{C O}}{4 \pi R^{2} \sigma_{S B}}\right)^{0.25}\right)$ whose results are presented in Table 1 and in appendix A.

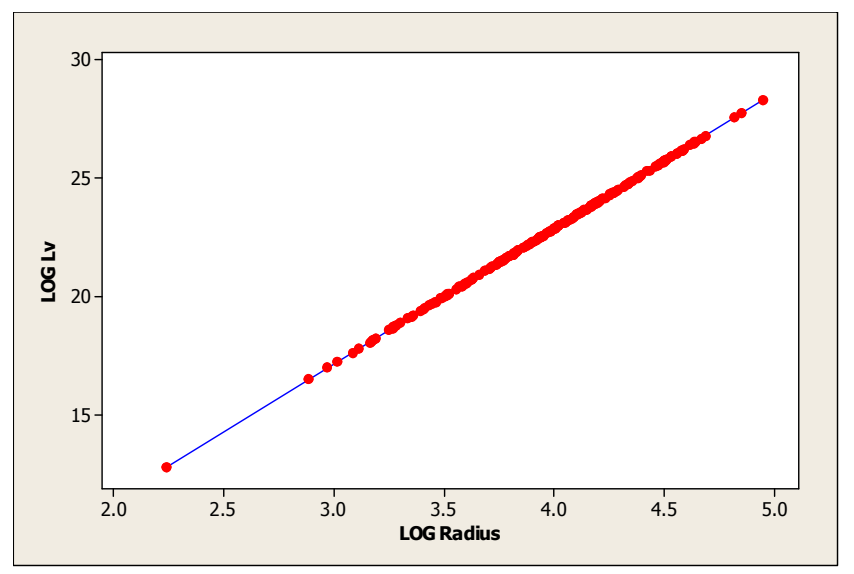

Figure 6. The scatter plot of Luminosity vs Radius of the selected GMCs in Milky Way.

Finally, it was also found that $\sum_{g}, M_{\star}$ and $L_{v}$ have a direct relationship and can be determined from the given correlations, (i.e. they are in direct proportion to each other).

On the search to know why the observed star formation efficiency is so low led comparing the above results with the results of $[4,20]$ and concluded that the reasons why the observed star formation efficiency is so low are: magnetic field, supersonic turbulence, self-regulation and Variations in the initial kinematic state of the cloud or star-forming region.

\section{Conclusion}

The overall efficiency with which the Milky Way GMC form stars has been estimated from our derived equation and the factor responsible for low SFE of Milky Way GMC have been outlined. One can conclude that: the $\epsilon_{G M C}$ can be calculated/estimated, if the average $\left(\Sigma_{\star}\right)$ and the $\sum_{g}$ of the cloud are known (which is present as $\epsilon_{G M C} \equiv$ $\Sigma_{\star} / \Sigma_{g}$ ) other than the relation given $[16,22]$, the $\epsilon_{G M C}$ declined as the $M_{g}$ increases and increases as the $\sum_{g}$ increases, the $M_{g}$ determines the collapse of cloud as described by Jean 1902, the low $\epsilon_{G M C}$ lies upon the densities of the GMCs as it determines the state of the cloud, the unbound states of internal structure are caused by magnetic field, supersonic turbulence and selfregulation, and finally, this density determines 1 . the number of stars that will be formed over the life time of the GMC 2. the state of cloud's internal structure (whether a cloud is in bound state or in unbound state (i.e., anything that can affect the density of cloud automatically can also affect the efficiency of that cloud)).

\section{Contributions to the General Knowledge}

The importance of star formation efficiency cannot be over emphasized. Yet the general problem of astronomers is no longer how stars form, but what mass fraction of a gas cloud can be converted into stars, what controls the star formation efficiency and reason why the observed efficiencies of GMCs are low.

This study on Milky Way GMCs has shown that unbound state of the internal structure determined by the density of GMCs caused by magnetic field, supersonic turbulence and self-regulation are the core mechanisms behind the observed low star formation efficiency of the Milky Way GMCs. And finally, that one can make better estimate of star formation efficiency using density or our derived equation.

\section{References}

[1] Burkhart B. \& Philip M., (2019), the self-gravitating gas fraction and the critical density for star formation, arXiv: $805.11104 v 3$ [astro-ph. GA].

[2] Clark, P. C., Bonnell I. A., (2004). Star formation in transient molecular clouds MNRAS, 347, L36.

[3] Clark, P. C., Bonnell I. A., Zinnecker H., Bate M. R., (2005). Star formation in unbound giant molecular clouds: the origin of OB associations MNRAS, 359.

[4] Clark, P. C.; Bonnell I. A. and Klessen R. S., (2008). The star formation efficiency and its relation to variations in the initial mass function MNRAS, 386, 3-10.

[5] Crutcher, R. M. (1999). Magnetic fields in molecular clouds: observations confront theory. Astrophys. J. 520, 706-713. doi: $10.1086 / 307483$.

[6] Crutcher, R. M., Wandelt, B., Heiles, C., Falgarone, E., and Troland, T. H. (2010). Magnetic fields in interstellar clouds from zeeman observations: inference of total field strengths by Bayesian analysis. Astrophys. J. 725, 466-479. doi: 10.1088/0004-637X/725/1/466.

[7] Cunningham A. J., Krumholz M. R., Mckee C. F., 2018, The effects of magnetic fields and protostellar feedback on lowmass cluster formation MNRAS 476, 771-792.

[8] Dame, T. M., Hartmann, D., \& Thaddeus, P. (2001). The Milky Way in molecular clouds: a new complete CO survey ApJ, 547, 792.

[9] Elmegreen, B. G., (2002). Star Formation from Galaxies to Globules ApJ, 577, 206.

[10] Grudic M. Y., Hopkins P. F., Faucher-Gigu ' ere C.-A., Quataert E., Murray 'N., Keres D., 2018, 'MNRAS, 475, 3511

[11] Grudic M. Y., Hopkins P. F., Lee E. J., Murray N., FaucherGiguere C. - A., Johnson L. C., 2019, MNRAS, 488 15011518 .

[12] Hennebelle P. and Inutsuka S. (2019) The Role of Magnetic Field in Molecular Cloud Formation and Evolution. Front. Astron. Space Sci. 6: 5. doi: 10.3389/fspas.2019.00005. 
[13] Krumholz M. R and Federrath C (2019) The Role of Magnetic Fields in Setting the Star Formation Rate and the Initial Mass Function. Front. Astron. Space Sci. 6: 7. doi: 10.3389/fspas.2019.00007.

[14] Kravtsov, A. V., (2003). On the origin of the global Schmidt law of star formation ApJ, 590, L1.

[15] Lee, E. J., Murray N., Rahman M., (2012). Milky way starforming complexes and the turbulent motion of the Galaxy's molecular gas ApJ, 752, 146.

[16] Lee, E. J., Miville-Deschenes M.-A., Murray N. W., (2016). Observational evidence of dynamic star formation rate in milky way giant molecular clouds ApJ, 833, 229.

[17] Li H., Vogelsberger M., Marinacci F., Gnedin O. Y., (2019), MNRAS 487, 364-380.

[18] Matzner, C. D., \& McKee, C. F., (2002) Efficiencies of LowMass Star and Star Cluster. ApJ, 545: 364-378.

[19] Mckee C. F., Ostriker E. C., (2019), Theory of star formation. Annu. Rev. Astro. Astrophys.
[20] Mo, H. J.; Vanden Bosch, F. C. \& White, S. D. M., (2010). Galaxy formation and evolution, MNRAS, 284, 192.

[21] Murray, N., \& Rahman, M. (2010). Star formation in massive clusters via the Wilkinson Microwave Anisotropy Probe and the Spitzer Glimpse survey ApJ, 709, 424.

[22] Murray, N. (2011). Star formation efficiencies and lifetimes of giant molecular clouds in the Milky Way ApJ, 729, 133.

[23] Plume, R., Jaffe D. T., Evans II N. J. Martin-Pintado J. AND Gomez-Gonzalez J. (1997). Dense gas and star formation: Characteristics of cloud cores associated with water masers ApJ 476: 730-749.

[24] Stahler, S. W. \& Palla, F. (2004). The Formation of Stars. Weinheim: Wiley-VCH. ISBN 3-527-40559-3.

[25] Williams, J. P., \& McKee, C. F. (1997). The Galactic distribution of $O B$ associations in molecular clouds. ApJ, 476, 166. 